\title{
Caracterización citogenética de Caesalpinia spinosa de los distritos de Tarma y Palca (Junín)
}

\author{
Cytogenetic characterization of Caesalpinia spinosa from Tarma and Palca (Junín)
}

\begin{abstract}
Alberto López ${ }^{1}$, María Siles-Vallejos ${ }^{1}$, Diego Orihuelaㄹ, José Linares', Shary Ríos ${ }^{1}$, Yvette Villafani ${ }^{1}$, Misael Guevara², Olga Bracamonte ${ }^{2}$
\end{abstract}

1 Laboratorio de Genética. Facultad de Ciencias Biologicas. Universidad Nacional Mayor de San Marcos. Lima, Perú.

2 Laboratorio de Citogenética, Facultad de Ciencias Biológicas, Universidad Nacional Mayor de San Marcos. Apartado 110058, Lima 11, Perú.

Email Alberto López: alopezs@unmsm.edu.pe

Email María Siles: mariansi@hotmail.com

Email Diego Orihuela: diego.orihuela tacuri@hotmail.com

Email José Linares: jrlinaresgonzales@gmail.com

Email Shary Ríos: shary49cam@hotmail.com

Email Yvette Villafani: yv.villafanib@yahoo.es

Email Misael Guevara: mguevarap@unmsm.edu.pe

Email Olga Bracamonte: obracamonteg@hotmail.com

Citación:

LópezA., M. Siles-Vallejos, D. Orihuela, J. Linares, S. Ríos, Y. Villafani, M. Guevara, O. Bracamonte. 2013. Caracterización citogenética de Caesalpinia spinosa de los distritos de Tarma y Palca (Junín). Rev. peru. biol. número especial 20(2): 245 - 248 (Marzo 2014)

\section{Resumen}

Se analizan los cromosomas somáticos de Caesalpinia spinosa (Feuillée ex Molina) Kuntze "Tara", de poblaciones silvestres de las localidades de Huinco y Palca (Junín). La especie es diploide $(2 n=24)$. Los cromosomas son de pequeño tamaño. Aunque los cariotipos de ambas localidades muestran el mismo número cromosómico, se encontraron diferencias en los parámetros morfológicos de los mismos, siendo la fórmula cariotípica para la localidad de Huinco: $6 m+6$ sm y para la localidad de Palca: $5 m+7$ sm.

Palabras clave: cromosoma; submetacéntrico; metacéntrico; metafase; cariotipo.

\section{Abstract}

Somatic chromosomes of Caesalpinia spinosa (Feuillée ex Molina) Kuntze, "Tara”, wild populations of Huinco and Palca (Junín) regions were studied. The specie were diploid $(2 n=24)$. Chromosomes were small. The karyotypes showed the same chromosome number, they found differences in morphological parameters of the same, with the karyotype formula for the town of Huinco: $6 m+6 \mathrm{sm}$ and the town of Palca: $5 m+7 \mathrm{sm}$.

Keywords: chromosome; submetacentric; metacentric; metaphase; karyotype.

\section{Introducción}

La "tara” (Caesalpinia spinosa (Feuillée ex Molina) Kuntze) es una especie nativa del Perú, que presenta sustancias útiles en las industrias farmacéutica, alimentaria, cosmética entre otras. Sin embargo, a pesar de su importancia no se cuenta aún con su caracterización cromosómica.

La citogenética es una herramienta muy útil para determinar esterilidad, reducción de la fertilidad, muerte embrionaria, caracterización de especies, variedades y razas, estudios filogenéticos, estudios ecotoxicológicos, entre otros. Asimismo, los análisis cariotípicos y citogenéticos han aportado de manera significativa tanto en la clasificación taxonómica como en el estudio evolutivo de especies vegetales que se desarrollan a diferentes altitudes (Baeza 2009).

El objetivo del presente trabajo fue analizar y comparar características citogenéticas de C. spinosa silvestres de las localidades de Huinco, distrito de Tarma; y Palca, distrito de Palca.

\section{Materiales y métodos}

La colecta del material biológico se realizó en poblaciones no cultivadas de C. spinosa de la provincia de Tarma, Región Junín, en las localidades de Huinco (3140 m de altitud), perteneciente al distrito de Tarma, y Palca (2791 m de altitud), ubicada en el distrito de Palca; colectándose vainas de Caesalpinia spinosa de cinco árboles por localidad y 8 vainas por árbol. Se entregaron ejemplares al Museo de Historia Natural de la Universidad Nacional Mayor de San Marcos para su identificación y deposito (0152-USM-2011). 

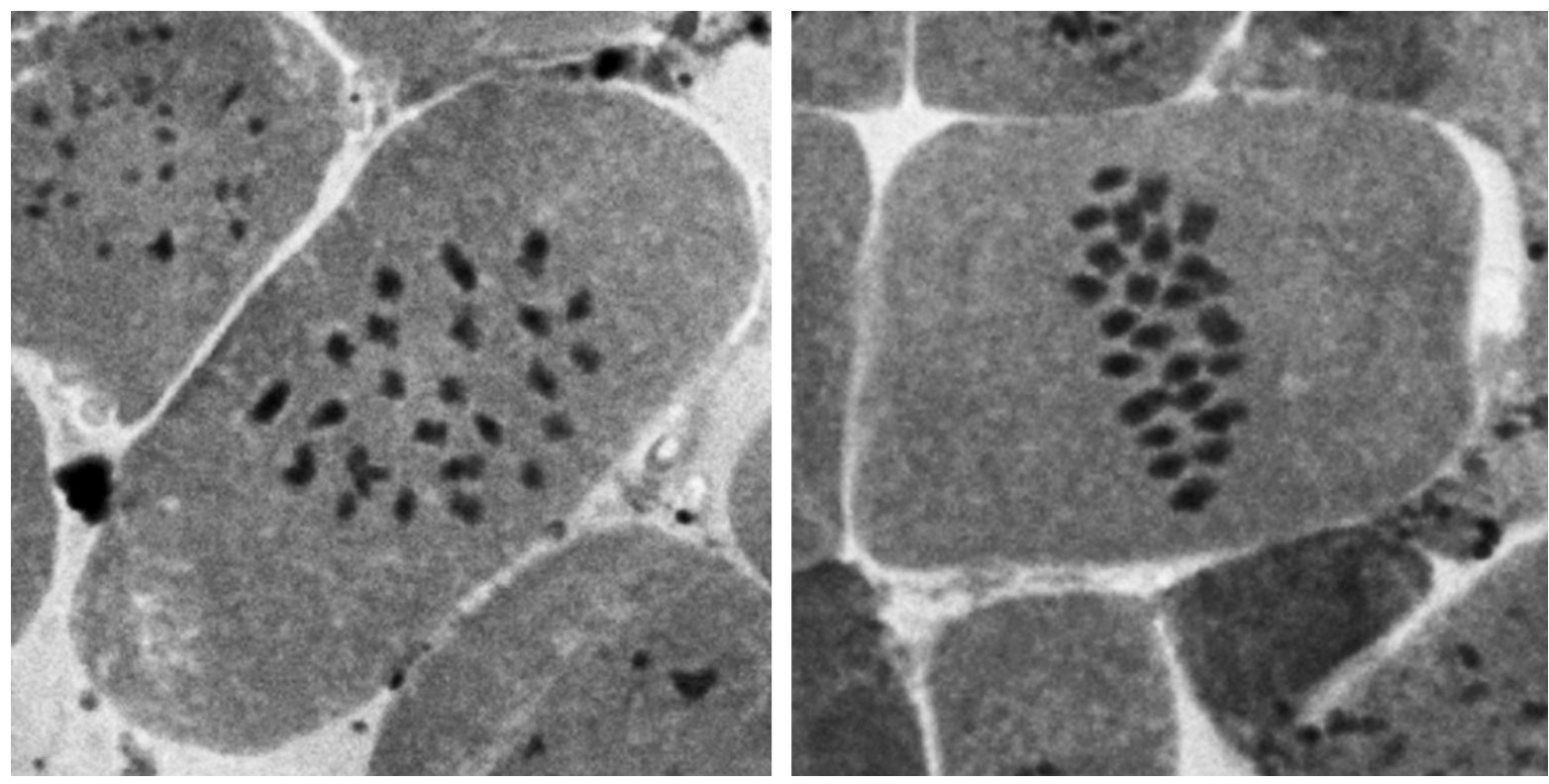

Figura 1 a y b. Metafases, localidad Palca, $2 n=24$ cromosomas.

Para el análisis citogenético, se escogieron 33 semillas al azar por cada localidad, las que se sometieron a un proceso de escarificación durante la noche inmediatamente después de la recolección. Luego se hicieron germinar las semillas hasta obtener raíces de $1 \mathrm{~cm}$ de longitud, las cuales se fijaron en etanol-ácido acético (3:1), se sumergieron en una solución de $\mathrm{HCl} 1 \mathrm{~N}$ para limpiar el citoplasma, se ablandaron en una solución Targa (acido acético, ácido láctico y agua destilada en proporciones 9:5:6) y, finalmente, se tińeron con orceína lacto acética $2 \%$ seguido del squash para la obtención de las placas metafásicas mitóticas. Se fotografiaron las mejores placas metafásicas, utilizándose un microscopio con cámara fotográfica incorporada, a un aumento de 1000X. Se realizó el análisis de las microfotografías para determinar el número y morfología cromosómica. Para determinar el tamaño de los cromosomas se hicieron las mediciones relativas de cada uno de ellos y se considero el promedio para así poder generalizar. Se utilizaron 15 placas metafasicas de cada localidad para el análisis. La clasificación de los cromosomas se hizo siguiendo la nomenclatura de Levan (1964).

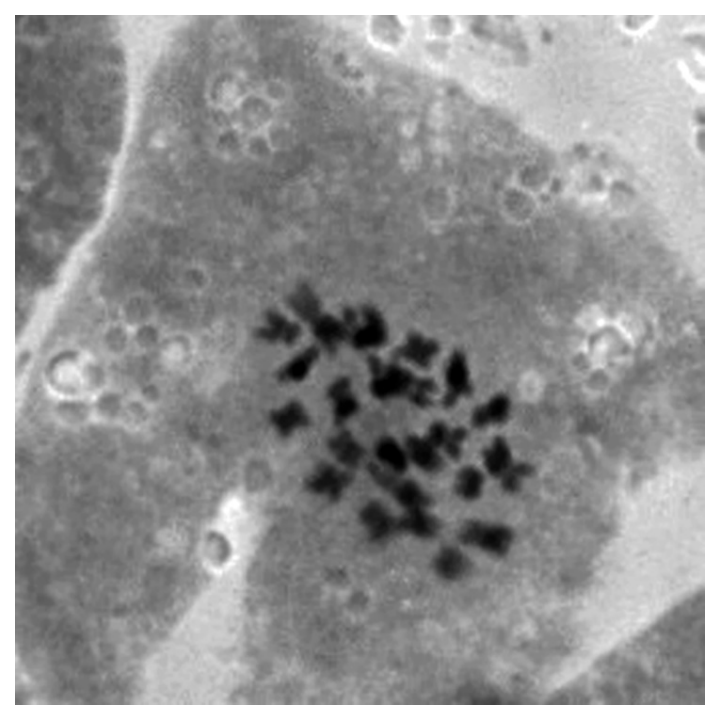

Figura 2. Metafase localidad de Huinco, $2 n=24$ cromosomas

\section{Resultados}

El número diploide hallado para Caesalpinia spinosa fue de $2 \mathrm{n}=24$ para ambas localidades (Figs 1 y 2 ). Sin embargo, a pesar de ser diploides, hemos observado algunas células poliploides (Fig 3). Los cromosomas son de pequeńo tamaño (Tablas 1 y 2), con una longitud relativa que varía de 0.46 a 0.87 para Huinco y de 0.52 a 1.16 para la localidad de Palca. Se observaron diferencias en la morfología de los cromosomas; encontrándose que en la localidad de Huinco el complemento presenta 6 pares metacéntricos y 6 pares submetacéntricos $(6 \mathrm{~m}+6 \mathrm{sm})$, siendo los pares $1,3,4,8,10$ y 12 metacéntricos y los pares 2, 5, 6, 7, 9 y 11 submetacentricos (Tabla 1, Fig 4); en tanto que en la localidad de Palca el complemento tiene 7 pares metacéntricos y 5 pares submetacéntricos $(7 \mathrm{~m}+5 \mathrm{sm})$, siendo los pares 1,2 , $5,7,10,11,12$ metacéntricos y los pares 3, 4, 6, 8 y 9 son submetacéntricos (Tabla 2, Fig 5).

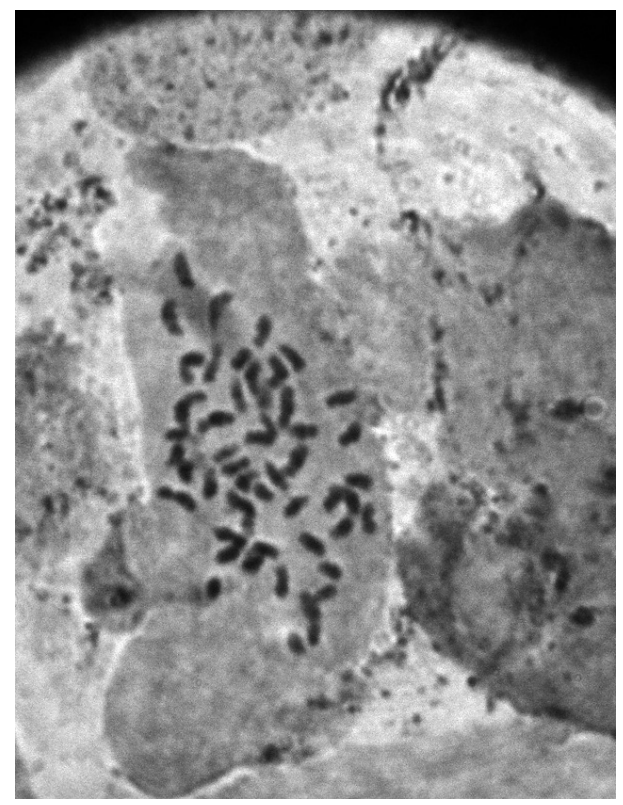

Figura 3. Placas metafásicas evidenciando poliploidía. 
Tabla 1. Morfología cromosómica de la localidad de Huinco. Longitudes relativas de $x p=$ longitud del brazo $p ; x q=$ longitud del brazo q; c = centrómero; IPC = índice de proporcionalidad centromérica.

\begin{tabular}{cccccc}
\hline PAR & $\mathbf{x p}$ & $\mathbf{x q}$ & $\mathbf{c}$ & $\mathbf{I P C}$ & TIPO \\
\hline $\mathbf{1}$ & 0.34 & 0.53 & 0.87 & 0.39 & $\mathrm{~m}$ \\
$\mathbf{2}$ & 0.25 & 0.51 & 0.76 & 0.32 & $\mathrm{sm}$ \\
$\mathbf{3}$ & 0.29 & 0.44 & 0.73 & 0.39 & $\mathrm{~m}$ \\
$\mathbf{4}$ & 0.31 & 0.36 & 0.67 & 0.46 & $\mathrm{~m}$ \\
$\mathbf{5}$ & 0.21 & 0.40 & 0.61 & 0.34 & $\mathrm{sm}$ \\
$\mathbf{6}$ & 0.22 & 0.37 & 0.59 & 0.37 & $\mathrm{sm}$ \\
$\mathbf{7}$ & 0.18 & 0.37 & 0.55 & 0.32 & $\mathrm{sm}$ \\
$\mathbf{8}$ & 0.25 & 0.30 & 0.55 & 0.45 & $\mathrm{~m}$ \\
$\mathbf{9}$ & 0.18 & 0.36 & 0.54 & 0.33 & $\mathrm{sm}$ \\
$\mathbf{1 0}$ & 0.25 & 0.28 & 0.53 & 0.47 & $\mathrm{~m}$ \\
$\mathbf{1 1}$ & 0.17 & 0.32 & 0.49 & 0.34 & $\mathrm{sm}$ \\
$\mathbf{1 2}$ & 0.18 & 0.28 & 0.46 & 0.39 & $\mathrm{~m}$ \\
\hline
\end{tabular}

Tabla 2. Morfología cromosómica de la localidad de Palca. Valores relativos, $x p=$ longitud del brazo $p ; x q=$ longitud del brazo $q ; c=$ centrómero; IPC = índice de proporcionalidad centromérica.

\begin{tabular}{cccccc}
\hline PAR & $\mathbf{x p}$ & $\mathbf{x q}$ & $\mathbf{c}$ & $\mathbf{I P C}$ & TIPO \\
\hline $\mathbf{1}$ & 0.57 & 0.59 & 1.16 & 0.49 & $\mathrm{~m}$ \\
$\mathbf{2}$ & 0.40 & 0.61 & 1.01 & 0.40 & $\mathrm{~m}$ \\
$\mathbf{3}$ & 0.29 & 0.58 & 0.87 & 0.33 & $\mathrm{sm}$ \\
$\mathbf{4}$ & 0.29 & 0.54 & 0.83 & 0.35 & $\mathrm{sm}$ \\
$\mathbf{5}$ & 0.36 & 0.43 & 0.79 & 0.40 & $\mathrm{~m}$ \\
$\mathbf{6}$ & 0.29 & 0.51 & 0.80 & 0.36 & $\mathrm{sm}$ \\
$\mathbf{7}$ & 0.29 & 0.48 & 0.77 & 0.38 & $\mathrm{~m}$ \\
$\mathbf{8}$ & 0.27 & 0.48 & 0.75 & 0.36 & $\mathrm{sm}$ \\
$\mathbf{9}$ & 0.27 & 0.47 & 0.74 & 0.36 & $\mathrm{sm}$ \\
$\mathbf{1 0}$ & 0.28 & 0.45 & 0.73 & 0.38 & $\mathrm{~m}$ \\
$\mathbf{1 1}$ & 0.28 & 0.37 & 0.65 & 0.43 & $\mathrm{~m}$ \\
$\mathbf{1 2}$ & 0.24 & 0.28 & 0.52 & 0.46 & $\mathrm{~m}$ \\
\hline
\end{tabular}

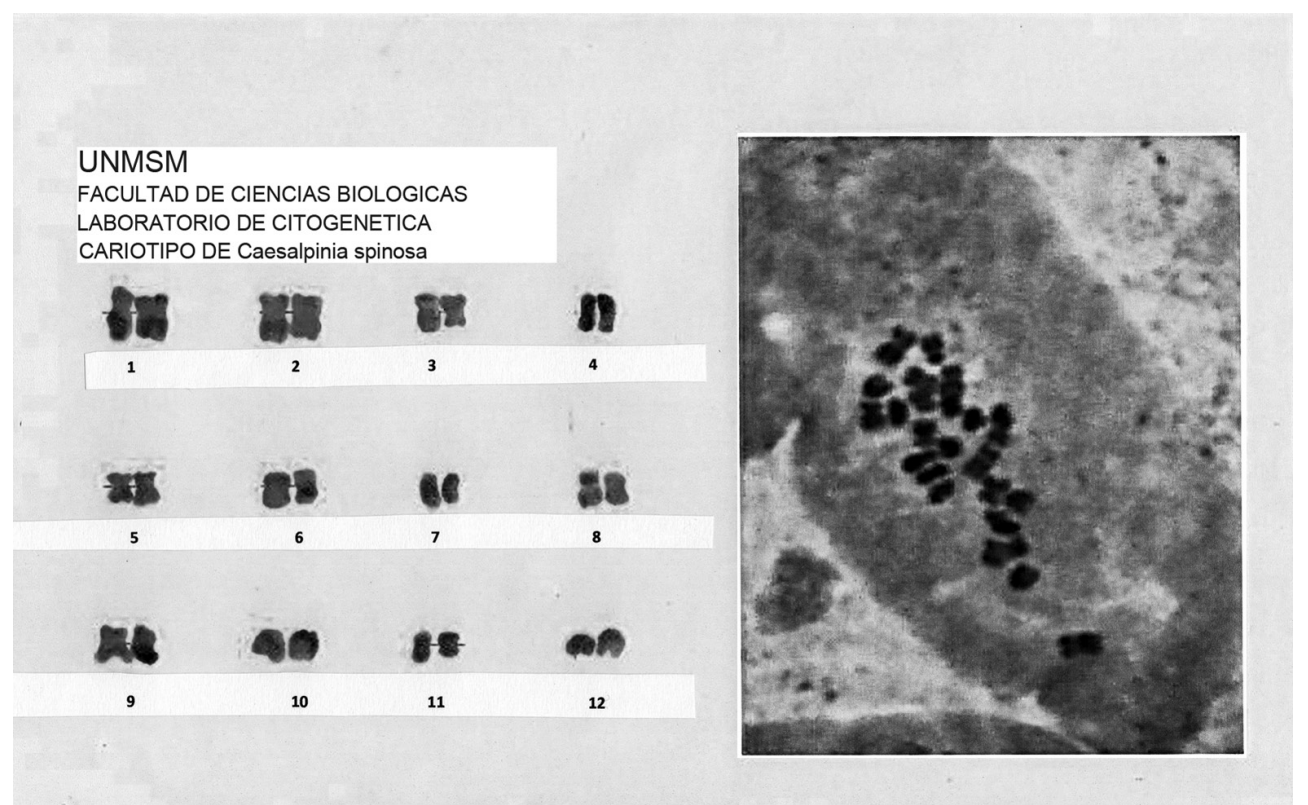

Figura 4. Cariotipo localidad de Huinco

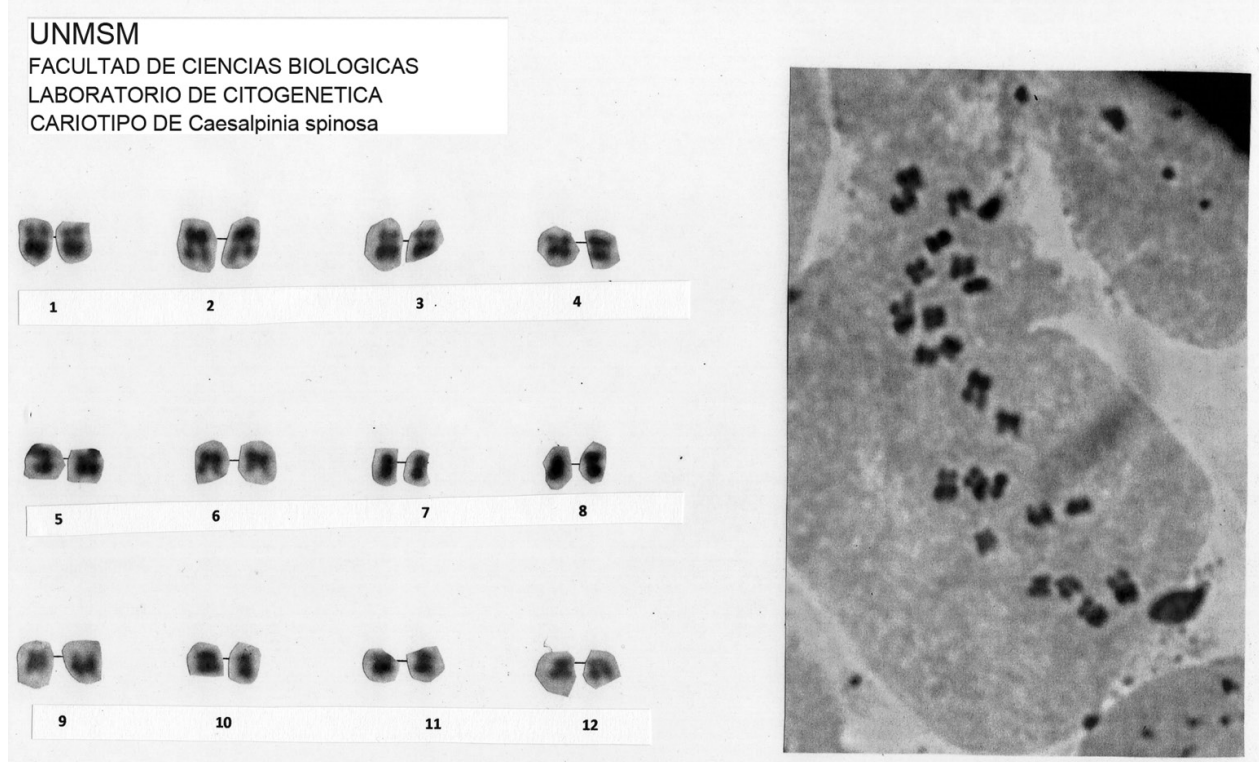

Figura5. Cariotipo de la localidad de Palca 


\section{Discusión}

Ha sido reportado que las especies de Caesalpinoideae presentan una gran variación en el número somático, con $2 \mathrm{n}=14,16$, 20, 22, 24, 26 y 28 cromosomas; existiendo series poliploides para el caso del género Caesalpinia ( $2 \mathrm{n}=12$ y $2 \mathrm{n}=24$ ) (Poggio et al. 2008). En nuestro caso, para las localidades de Huinco y Palca, hemos encontrado $2 \mathrm{n}=24$ cromosomas, este número de cromosomas coincide con el que presenta la mayoría de las especies del género las cuales muestran $\mathrm{x}=12$. Silva et al (2012) describen la morfologia cromosómica de cinco especies de Caesalpinia del Brasil, reportando un numero diploide de 24, nuestras observaciones son concordantes con el mismo valor. Las especies de Caesalpinia que han sido estudiadas citogenéticamente tienen cromosomas pequeńos (Cangiano \& Bernardello 2005, Zanin \& Cangiano 2001) lo que concuerda con nuestras observaciones. La fórmula cariotípica muestra únicamente cromosomas metacécntricos y submetacéntricos (m y sm), lo cual es muy común dentro de la subfamilia Caesalpinioidae (Souza et al, 2004).

Gómez et al. (2003) señalan que el análisis de cariotipos entre poblaciones de plantas de la misma especie, desarrolladas en áreas distintas, puede ayudar a entender los aspectos evolutivos, ecológicos y de adaptación. En nuestro estudio, el análisis citogenético de las dos poblaciones estudiadas revela diferencias en el cariotipo; aunque ambas poblaciones presentan $2 n=24$ cromosomas y en ambos casos los complementos están compuestos por cromosomas metacéntricos y submetacéntricos, encontramos diferencias en cuanto al número de metacéntricos y submetacéntricos. Así, en la localidad de Huinco la fórmula es $6 m+6 s m$ mientras que en Palca la fórmula cariotípica es $7 \mathrm{~m}+5 \mathrm{sm}$ evidenciando la presencia de dos citotipos diferentes. Encontramos que hay polimorfismo interpoblacional en los cromosomas 2,3,4,5,7,8 y 11 entre ambas localidades. Nuestras observaciones indican que a pesar de poseer el mismo nivel de ploidía, existen diferencias en los parámetros morfológicos del cariotipo, lo que indica que el cariotipo de C. spinosa no es uniforme entre poblaciones. Al respecto, Tapia-Pastrana et al. (2012) refieren que la posibilidad de encontrar citotipos con formulas cromosomicas diferentes estaría relacionada con la distribución ecológica y geográfica de la planta y que la divergencia en los genomas ocurriría como una consecuencia adaptativa a condiciones ambientales específicas. Esto mismo ha sido observado en otras monocotiledoneas como Alstroemeria hookeri L. asi como en Crotalaria (Fabaceae) (Baeza et al. 2006).

\section{Agradecimientos}

El presente trabajo fue financiado por el Vice rectorado de Investigación de la UNMSM como parte del Proyecto Multidisciplinario PEM 2009B03.

\section{Literatura citada}

Baeza C. M., O. Schrader, E. Ruiz. \& Negritto M. 2006. Análisis comparativo del cariotipo en poblaciones de Alstroemeria ligtu subsp ligtu y A. ligtu subs. siimsi (Alstroemeriaceae) de Chile. Darwiniana 44 (2): 313-318.

Baeza C., E. Ruiz \& M. A. Negritto. 2009. Importancia del cariotipo en la taxonomía y evolución del género Chaetanthera (asteraceae): evidencias preliminares para especies que crecen en Chile. Gayana Bot. 66(1): 50-57, 2009

Cangiano M. A. and G. Bernardello. 2005. Karyotype analysis in Argentinean species of Caesalpinia (Leguminosae). Caryologia, Vol. 58, nº 3:262268. DOI: 10.1080/00087114.2005.10589461

Gómez-Acevedo S.L. \& F. Tapia-Pastrana. 2003. Estudio genecológico en Prosopis laevigata, Acacia farnesiana y Acacia schaffneri (leguminosae). Darwiniana 41(1-4): 47-54

Levan A., K. Fredga \& A. Sandberg. 1964. Nomenclature for centromeric position on chromosomes. Hereditas, 52: 201-220. DOI: 10.1111/j.1601-5223.1964.tb01953.x

Silva P., M. Magalhaes, \& R. Xavier. 2012. Karyomorphology of Caesalpinia Species (Caesalpinioideae: Fabaceae) from Caatinga and Mata Atlantica Biomes of Brazil. Journal of Plant Studies, 1: 82-91. DOI: 10.5539/jps.v1n2p82

Souza M. G. C. and A. M. Benko-Iseppon. 2004. Cytogenetics and chromosome banding patterns in Caesalpinioideae and Papilionioideae species of Pará, Amazonas, Brazil. Botanical Journal of the Linnean Society, 144, 181-191. DOI: 10.1111/j.1095-8339.2003.00230.x

Poggio L., S.M. Espert \& R.H. Fortunato. 2008. Citogenética evolutiva en Leguminosas americanas. Rodriguésia 59 (3): 423-433.

Tapia-Pastrana F., P. Mercado-Ruaro \& S. Gómez-Acevedo. 2012.Contribución a la citogenética de Tamarindus indica (Leguminosae: Caesalpinioideae). Act. Bot. Mex. 98: 99-110.

Zanin L. \& M. Cangiano. 2001. El cariotipo de Hoffmannseggia glauca (FABACEAE). Darwiniana 39 (1-2): $11-13$. 